\title{
Formación de los profesionales de la salud en un contexto asistencial con uso de las tecnologías de la información y la comunicación
}

\author{
Jordi Galimany-Masclans, Eva Garrido-Aguilar, Joan M. Estrada-Masllorens, M. Rosa Girbau-García
}

\begin{abstract}
Resumen. La enfermera debería incorporar nuevas habilidades en el manejo de las tecnologías de la información y la comunicación (TIC) que faciliten el desarrollo de su profesión. En este proceso se plantean dudas y resistencias que pueden convertirse en barreras para el desarrollo de su labor profesional. Las estrategias para vencer resistencias ante las nuevas formas de trabajar pasan por facilitar una formación correctamente planificada y adaptada a los profesionales que deben enfrentarse a estas nuevas tecnologías. Algunos de los aspectos clave relacionados con la formación de las enfermeras ante nuevas competencias y que pueden contribuir a su desarrollo exitoso en los centros asistenciales son la definición de tácticas claras de formación con identificación de buenas prácticas asistenciales como mecanismo conductor, el apoyo a las enfermeras asistenciales por parte de las gestoras que lideran los equipos asistenciales y la identificación de la predisposición de los usuarios frente a la formación. Lograr el éxito en el uso de las TIC puede residir en la participación activa de las propias enfermeras en el proceso formativo planificado, teniendo en cuenta nuevos enfoques asistenciales por vía telemática, como alternativa al actual modelo asistencial, tradicionalmente presencial.
\end{abstract}

Palabras clave. Enfermería. Formación. Tecnologías de la información y la comunicación.

\section{Training health professionals within a healthcare context using information and communication technologies}

Summary. The nurse should incorporate new skills in the management of information technology and communication (ICT) to facilitate the development of their profession. This process may raise doubts and resistances that can become barriers when developing their work. Thus strategies to overcome resistance to new ways of working undergo training facilitate well planned and tailored to the professionals who must cope with new technologies. Some of the key issues related to the education of nurses related to new skills, are the definition of clear strategies with training support identifying best practices as mechanism driver training, support to practicing nurses by the leaders who lead care teams as well as the identification of the willingness of users from training. Achieving success in the use of ICT in the active participation of nurses themselves planned in the learning process, taking into account new approaches of tele-care, as an alternative of traditional care.

Key words. Information and communication technology. Nursing. Training.

\section{Contexto asistencial}

Los profesionales de la salud se enfrentan a continuos avances derivados de la incorporación de nuevos recursos sanitarios. Las tecnologías de la información y la comunicación (TIC) son, en este momento, una herramienta clave en los centros asistenciales. Es evidente que los profesionales de la salud en general, y las enfermeras en particular, están acostumbrados a la incorporación de nuevas técnicas, nuevos protocolos y, en definitiva, nuevas maneras de llevar a cabo su labor asistencial. Esta realidad es aceptada por las enfermeras como un elemento natural en su desempeño profesional [1].
No obstante, la puesta en marcha de las TIC en los sistemas sanitarios es un proceso complejo que requiere muchos esfuerzos, a la vez que supone retos importantes para los profesionales y presenta oportunidades de mejora para las instituciones, los profesionales de la salud y los usuarios. En la actualidad, las enfermeras están sometidas a una elevada presión asistencial con un aumento de las exigencias de la población, junto con la escasez de recursos humanos y materiales y la necesidad de control del gasto, lo que pone de manifiesto la necesidad de acompañar a los profesionales en la incorporación de estos instrumentos con el objetivo de ver compensado su esfuerzo. Como en todo proceso de cam-
Escuela Universitaria de Enfermería. Universitat de Barcelona. L'Hospitalet de Llobregat, Barcelona, España.

Correspondencia:

Prof. Jordi Galimany Masclans. Departamento de Enfermería de Salud Pública, Salud Menta y Materno-Infantil. Campus de Bellvitge. Pavelló de Govern, 3. ㄹ planta. Feixa Llarga, $\mathrm{s} / \mathrm{n}$. E-08907 L'Hospitalet de Llobregat (Barcelona).

E-mail:

jordigalimany@ub.edu

Conflicto de intereses: No declarado.

Competing interests: None declared.

(c) 2013 FEM 
bio, hay que tener en cuenta el factor humano como elemento crucial, ya que es el que debe garantizar un nivel de eficacia que permita avanzar en la implantación de las TIC $[2,3]$.

La atención de salud que reciben las personas por parte de los sistemas sanitarios tradicionalmente se ha caracterizado por ser presencial, acudiendo éstas a la consulta para cualquier tipo de demanda asistencial, ya sea de carácter curativo, de control o seguimiento de un proceso crónico o de algún programa de prevención y cribado. En estos momentos, la situación económica de los sistemas sanitarios plantea la necesidad de buscar alternativas a la atención sanitaria con un enfoque presencial. Estas alternativas parecen encaminarse hacia modelos y sistemas de asistencia y prestación de cuidados virtuales o no presenciales. Son ejemplos de ello la atención telefónica, la atención vía web o la teleasistencia o teleenfermería.

Parece evidente que en este nuevo panorama asistencial, en el que la enfermera es una de las piezas clave por su proximidad y el profesional de referencia para el usuario, ésta puede ser uno de los profesionales sanitarios que vea más modificada su manera de prestar los cuidados al paciente. Estos cambios plantean ventajas e inconvenientes $y$, lo que es más importante, nuevos retos para la enfermera que deberá adaptarse a esta situación, formándose y aprendiendo cuáles son las claves de este nuevo contexto de prestación de cuidados y entendiendo que supone un cambio de paradigma en su rol de cuidadora.

En este proceso de integración de las TIC en el ámbito asistencial no existe vuelta atrás, ya que cada vez más el sistema y el propio usuario entienden que la asistencia puede ser no presencial o virtual, manteniendo la misma excelencia en los cuidados. Al mismo tiempo, las instituciones sanitarias deben comprender que la formación es un elemento imprescindible para afrontar con éxito este reto antes, durante y después de la incorporación en los sistemas asistenciales [4].

\section{Formación en un contexto asistencial con uso de las TIC}

La formación referida a las TIC debería llevarse a cabo durante el periodo de formación universitaria de los futuros profesionales, así como a lo largo del desarrollo profesional, ya que es necesaria una actualización constante de estas nuevas tecnologías, que evolucionan de forma continua. Para los profesionales, el nuevo escenario tecnológico supone adap- tarse a la atención de usuarios que disponen de acceso a la información sobre su salud de manera continua e ilimitada. En consecuencia, pueden generarse nuevas demandas para el sistema y para sus profesionales. Será imprescindible formar a los profesionales en el uso y conocimiento crítico de las herramientas TIC para que sean capaces de dar respuesta efectiva a las demandas de pacientes cada vez más informados y con mayores conocimientos sobre su salud. Por tanto, es básico que los profesionales de la salud asuman que el paciente dispondrá de más información y tendrá un papel más proactivo, con las ventajas e inconvenientes que esto puede suponer.

Analizar la incorporación y el uso de las TIC desde el punto de vista de la formación supone considerar la organización y sus interrelaciones, sus políticas y prácticas asistenciales y las barreras existentes. Durante el proceso de incorporación y consolidación del uso de las TIC, la formación es un aspecto central. Ante estos nuevos retos, los profesionales de la salud manifiestan dudas y resistencias que, en la mayoría de los casos, se pueden minimizar o paliar con una visión multidisciplinar, considerando la implementación de las TIC desde una perspectiva de cambio organizacional y diseñando una formación adecuada $[3,5]$.

En este sentido, diversos estudios ponen de manifiesto que aquello que más preocupa a los profesionales sanitarios en general, y a las enfermeras en concreto, en el uso de las TIC se relaciona especialmente con las habilidades y los conocimientos que se necesitan para su manejo y con factores que determinan su encaje en la organización de las instituciones sanitarias. Estos aspectos pueden actuar como barrera, pero pueden superarse mediante formación y la participación en el proceso de quienes deben utilizarlos. La formación, además de capacitar para el desempeño de sus competencias, facilita la implicación, la responsabilización y la participación de los profesionales que van a utilizar estos recursos [6-8].

Las TIC pueden facilitar los cuidados a la población en un contexto sanitario cambiante, con un incremento de pacientes crónicos con pluripatología, y en un contexto demográfico, asistencial y social de creciente complejidad y con dificultades para que el sistema sanitario sea equitativo, accesible y sostenible $[9,10]$.

Destaca, en este sentido, la falta de trabajos de evaluación centrados en la relación entre la formación de las enfermeras y las implementaciones exitosas de las TIC en los sistemas de salud. Seguramente esto se debe a que son procesos todavía re- 
cientes que se deberán analizar en profundidad a medida que estén más generalizados [4,6,11-14].

Diferentes autores plantean la necesidad de reforzar algunos aspectos relacionados con la formación de las enfermeras en el uso de las TIC, con el objetivo de mejorar la calidad asistencial y facilitar su implantación en los centros asistenciales. A continuación se enumeran algunas de las estrategias planteadas:

- La definición de estrategias claras de formación, con identificación de buenas prácticas clínicas o asistenciales como mecanismo conductor de dicha formación en relación con la implementación de los nuevos dispositivos [15].

- El desarrollo de recursos que respondan a las necesidades asistenciales y que exista conexión entre éstas y los servicios tecnológicos. Es decir, que la tecnología responda a las necesidades de los servicios y a las necesidades de sus profesionales para poder llevar a cabo la asistencia de salud a los ciudadanos [16].

- El diseño de herramientas fáciles de usar, que faciliten el aprendizaje por parte de las enfermeras asistenciales (que son los usuarios finales) y que tengan impacto relevante en los cuidados y en los pacientes. Que sean relevantes para ellos como herramienta útil para analizar sus riesgos de salud y que integren todos los pasos del proceso de cuidado o proceso asistencial $[17,18]$.

- El establecimiento de estrategias y medidas de apoyo para las enfermeras asistenciales por parte de las enfermeras que lideran y gestionan los equipos asistenciales. Toma de decisiones enfocadas al cuidado y no únicamente a la gestión de los recursos humanos o materiales [19-21].

- La identificación de la predisposición y cuáles son los aspectos de la herramienta destacados por los usuarios finales como elementos positivos. Por ejemplo, mediante la realización de una encuesta que recoja las inquietudes y los puntos débiles y puntos fuertes del proceso de incorporación de las nuevas tecnologías [22].

- La implicación, la capacitación, la participación y la responsabilización de los usuarios finales de la herramienta con el proceso de implementación y con formación planificada [23].

- La participación de los usuarios en el diseño de las herramientas [2,24].

- El despliegue de dispositivos que ayuden y faciliten el desarrollo de la práctica clínica de las enfermeras [25].

- La valoración y la consideración de la edad y nivel de desarrollo profesional de las enfermeras que usarán la herramienta [13].
Algunos proveedores de servicios de salud utilizan la figura de la enfermera como referencia de formación. El objetivo es disponer de una persona formada, con conocimiento avanzado, que puede actuar como asesora y acompañar al resto del equipo asistencial en los procesos de implementación de las TIC y en las habituales actualizaciones de éstas. Así se facilita que el equipo asistencial disponga de una figura próxima, formada y experta para sacar el máximo provecho de las actualizaciones y las nuevas aplicaciones de las herramientas, teniendo en cuenta además que actúan como filtro previo de dichas mejoras y actualizaciones.

Además de las estratégicas comerciales, algunos autores relacionan las barreras asociadas a la implementación de dichos recursos con aspectos no directamente asociados con el dispositivo en sí, sino con aspectos estructurales de los servicios o departamentos donde se implementa.

También la formación puede convertirse en una barrera si no se considera un aspecto clave en el proceso, puesto que además de ser necesaria para que se domine y se saque el máximo provecho a los recursos TIC, constituye un aspecto que actúa como mecanismo de incentivación y consolidación de las innovaciones que los profesionales sanitarios deben adquirir en su día a día asistencial [26,27].

\section{Conclusiones}

Ante los actuales retos tecnológicos que afrontan las enfermeras, es imprescindible explorar la formación que necesitan estos profesionales. Especialmente deberían diseñarse estrategias que potencien la avidez de conocimiento y consigan incentivar el uso de nuevas tecnologías o el desarrollo de nuevas competencias.

Si se desea dotar de valor añadido al modelo asistencial actual, debe mejorarse la capacidad innovadora de los cuidados ofrecidos a la población del siglo XXI.

Los aspectos o áreas considerados claves en relación a la formación se podrían agrupar en tres grandes apartados: el liderazgo, las prácticas o maneras de trabajar y las habilidades para el desarrollo y la gestión de la información. De manera más concreta, se puede hablar de lecciones aprendidas por aquellas instituciones que han llevado a cabo estos procesos de incorporación de las TIC. En ellas destaca, de manera relevante, la formación y su diseño para los profesionales, la conexión entre las necesidades y los servicios tecnológicos (es decir, que la tecnología responda a las necesidades planteadas 
en los servicios), y la aplicación y desarrollo de estándares de calidad. Además, todo ello debe contemplarse en un entorno de rediseño de los servicios y de la estructura de gobernanza integrada con la toma de decisiones basadas en evidencias fiables y cuantificables.

Bibliografía

1. Edwards G, Kitzmiller RR, Breckenridge-Sproat S. Innovative health information technology training: exploring blended learning. Comput Inform Nurs 2012; 30: 104-9.

2. Gruber D, Cummings GG, LeBlanc L, Smith DL. Factors influencing outcomes of clinical information systems implementation: a systematic review. Comput Inform Nurs 2009; 27: 151-63 [quiz 164-5].

3. Román-Viñas R. Factors of success in the implementation of the technologies of the information and the communication in the health systems. The human factor. Med Clin (Barc) 2010; 134 (Suppl 1): 39-44.

4. Gagnon MP, Desmartis M, Labrecque M, Legare F, Lamothe L, Fortin JP, et al. Implementation of an electronic medical record in family practice: a case study. Inform Prim Care 2010; 18: $31-40$.

5. Lluch M. Healthcare professionals' organisational barriers to health information technologies - a literature review. Int J Med Inform 2011; 80: 849-62.

6. Gagnon MP, Legare F, Labrecque M, Fremont P, Pluye P, Gagnon J, et al. Interventions for promoting information and communication technologies adoption in healthcare professionals. Cochrane Database Syst Rev 2009; 1: CD006093.

7. Ayatollahi H, Bath PA, Goodacre S. Paper-based versus computer-based records in the emergency department: staff preferences, expectations, and concerns. Health Informatics J 2009; 15: 199-211.

8. Timmons S. Nurses resisting information technology. Nurs Inq 2003; 10: 257-69.

9. Casalino LP. Analysis \& commentary. A Martian's prescription for primary care: overhaul the physician's workday. Health Aff (Millwood) 2010; 29: 785-90.

10. Shrager FE. Revamping end-user training. Comput Inform Nurs 2010; 28: 5-7.

11. Laramee AS, Bosek M, Kasprisin CA, Powers-Phaneuf T. Learning from within to ensure a successful implementation of an electronic health record. Comput Inform Nurs 2011; 29: 468-77 [quiz 478-9].

12. Kaelber DC, Jha AK, Johnston D, Middleton B, Bates DW. A research agenda for personal health records (PHRs). J Am Med Inform Assoc 2008; 15: 729-36.
13. Eley R, Fallon T, Soar J, Buikstra E, Hegney D. Barriers to use of information and computer technology by Australia's nurses: a national survey. J Clin Nurs 2009; 18: 1151-8.

14. Hart MD. Informatics competency and development within the US nursing population workforce: a systematic literature review. Comput Inform Nurs 2008; 26: 320-9 [quiz 330-1].

15. Makela K, Virjo I, Aho J, Kalliola P, Kurunmaki H, Uusitalo L, et al. Management of electronic patient record systems in primary healthcare in a Finnish county. Telemed J E Health 2010; 16: 1017-23.

16. Bostrom AC, Schafer P, Dontje K, Pohl JM, Nagelkerk J, Cavanagh SJ. Electronic health record: implementation across the Michigan Academic Consortium. Comput Inform Nurs 2006; 24: 44-52.

17. Ketikidis P, Dimitrovski T, Lazuras L, Bath PA. Acceptance of health information technology in health professionals: an application of the revised technology acceptance model. Health Informatics J 2012; 18: 124-34.

18. Schleyer RH, Burch CK, Schoessler MT. Defining and integrating informatics competencies into a hospital nursing department. Comput Inform Nurs 2011; 29: 167-73.

19. Yu P, Li H, Gagnon MP. Health IT acceptance factors in long-term care facilities: a cross-sectional survey. Int J Med Inform 2009; 78: 219-29.

20. McCartney PR. Leadership in nursing informatics. J Obstet Gynecol Neonatal Nurs 2004; 33: 371-80.

21. Wilhoit K, Mustain J, King M. The role of frontline RNs in the selection of an electronic medical record business partner. Comput Inform Nurs 2006; 24: 188-95.

22. Van der Meijden MJ, Tange H, Troost J, Hasman A. Development and implementation of an EPR: how to encourage the user. Int J Med Inform 2001; 64: 173-85.

23. Adam P, Permanyer-Miralda G, Sola-Morales O, CanelaSoler J. Information, knowledge and healthcare practice: professionals participation as the key element of the gear. Med Clin (Barc) 2010; 134 (Suppl 1): 10-5.

24. Stevenson JE, Nilsson GC, Petersson GI, Johansson PE. Nurses' experience of using electronic patient records in everyday practice in acute/inpatient ward settings: a literature review. Health Informatics J 2010; 16: 63-72.

25. Moen A. A nursing perspective to design and implementation of electronic patient record systems. J Biomed Inform 2003; 36: 375-8.

26. McAlearney AS, Robbins J, Hirsch A, Jorina M, Harrop JP. Perceived efficiency impacts following electronic health record implementation: an exploratory study of an urban community health center network. Int J Med Inform 2010; 79: 807-16.

27. Furukawa MF, Raghu TS, Shao BB. Electronic medical records, nurse staffing, and nurse-sensitive patient outcomes: evidence from the national database of nursing quality indicators. Med Care Res Rev 2011; 68: 311-31. 